\title{
Alterações no paladar advindos de quimioterapia convencional
}

\author{
Changes in taste resulting from conventional chemotherapy \\ Cambios en el gusto resultantes de la quimioterapia convencional
}

Recebido: 28/10/2021 | Revisado: 05/11/2021 | Aceito: 09/11/2021 | Publicado: 14/11/2021

Eduardo Henrique dos Santos Silva
ORCID: https://orcid.org/0000-0002-7685-8260
Faculdade Independente do Nordeste, Brasil
E-mail: eudardo.bdo@ gmail.com
Yuri Lopes de Aguiar
ORCID: https://orcid.org/0000-0002-8936-7970
Faculdade Independente do Nordeste, Brasil
E-mail: yuri.aguiar54@ gmail.com
Polyana Argolo Souza Amaral
ORCID: https://orcid.org/0000-0002-4742-5562
Faculdade Independente do Nordeste, Brasil
E-mail: polyana.argolo@ gmail.com
Lara Correia Pereira
ORCID: https://orcid.org/0000-0002-5529-9260
Faculdade Independente do Nordeste, Brasil
E-mail: lcp.larapereira @ gmail.com

\section{Resumo}

O câncer está ligado ao aumento desordenado de células, possui a capacidade de invadir tecidos vizinhos no local de origem e/ou instalar-se em órgãos distantes via corrente sanguínea. O tratamento antineoplásico pode gerar efeitos colaterais, na mucosa oral, ficando evidente através do surgimento da mucosite oral. Também podemos observar alterações no paladar, como: hipogeusia, disgeusia, ageusia e hipergeusia. O presente trabalho visa revisar a literatura acerca das alterações do paladar provocadas por 5-fluoruracila (5-FU), leucovorine, oxaliplatina, gencitabine, capecitabine, cisplatina, ciclofosfamida, doxorubicina, irinotecan e metotrexato, sendo medicamentos antineoplásicos convencionais. Este estudo foi realizado através do levantamento de dados no período de 2009 até 2021 , a partir da literatura científica na base de dados PubMed, Lilacs, Cochrane e Scielo em língua inglesa e espanhola, valendo-se das palavras chaves Antinéoplasicos; Paladar Distorcido; Disgeusia; Estomatite; Cavidade Oral; juntamente com os operadorer booleanos "and" e "or", de forma a combinar os descritores citados. Diante do exposto, os fármacos quimioterápicos convencionais afetam as células gustativas e os processos bioquímicos referentes a sua renovação celular, gerando alterações no paladar como hipogeusia, disgeusia, ageusia e hipergeusia.

Palavras-chave: Antineoplásicos; Paladar distorcido; Disgeusia; Estomatite; Cavidade oral.

\begin{abstract}
Cancer is linked to the disordered increase of cells, has the ability to invade neighboring tissues in the place of origin and/or install itself in distant organs via the bloodstream. Antineoplastic treatment can generate side effects in the oral mucosa, being evident through the appearance of oral mucositis. We can also observe changes in taste, such as: hypogeusia, dysgeusia, ageusia and hypergeusia. The present work aims to review the literature on taste alterations caused by 5-fluorouracil (5-FU), leucovorine, oxaliplatin, gemcitabine, capecitabine, cisplatin, cyclophosphamide, doxorubicin, irinotecan and methotrexate, being conventional antineoplastic drugs. This study was carried out by collecting data from 2009 to 2021, from scientific literature in the PubMed, Lilacs, Cochrane and Scielo databases in English and Spanish, using the key words Antineoplastics; Distorted taste; Dysgeusia; Stomatitis; Oral cavity; together with the Boolean operators "and" and "or", in order to combine the aforementioned descriptors. Given the above, conventional chemotherapy drugs affect taste cells and biochemical processes related to their cell renewal, generating changes in taste such as hypogeusia, dysgeusia, ageusia and hypergeusia.
\end{abstract}

Keywords: Antineoplastics; Distorted taste; Dysgeusia; Stomatitis; Oral cavity.

\section{Resumen}

El cáncer está relacionado con el aumento desordenado de células, tiene la capacidad de invadir los tejidos vecinos en el lugar de origen y / o instalarse en órganos distantes a través del torrente sanguíneo. El tratamiento antineoplásico puede generar efectos secundarios en la mucosa bucal, siendo evidentes por la aparición de mucositis bucal. También podemos observar cambios en el gusto, como: hipogeusia, disgeusia, ageusia e hipergeusia. El presente trabajo tiene como objetivo revisar la literatura sobre las alteraciones del gusto causadas por 5-fluorouracilo (5-FU), leucovorina, oxaliplatino, gemcitabina, capecitabina, cisplatino, ciclofosfamida, doxorrubicina, irinotecán y metotrexato, siendo 
fármacos antineoplásicos convencionales. Este estudio se llevó a cabo mediante la recolección de datos de 2009 a 2021, de la literatura científica en las bases de datos PubMed, Lilacs, Cochrane y Scielo en inglés y español, utilizando las palabras clave Antineoplásticos; Sabor distorsionado; Disgeusia; Estomatitis; Cavidad oral; junto con los operadores booleanos "y" y "o", con el fin de combinar los descriptores antes mencionados. Dado lo anterior, los fármacos quimioterápicos convencionales afectan las células gustativas y los procesos bioquímicos relacionados con su renovación celular, generando cambios en el gusto como hipogeusia, disgeusia, ageusia e hipergeusia.

Palabras clave: Antineoplásticos; Gusto distorsionado; Disgeusia; Estomatita; Cavidad oral.

\section{Introdução}

O câncer é uma doença ligada ao aumento desordenado quanti e qualitativamente das células, que possui a capacidade de invadir tecidos vizinhos no local de origem e/ou instalar-se em órgãos distantes via corrente sanguínea. Tal descontrole celular é proveniente de erros sucessivos na replicação do material genético presente no interior do núcleo das células (Rahnama et al., 2015).

O Instituto Nacional de Câncer (INCA) estima 309.750 e 316.280 novos casos no Brasil em homens e mulheres, respectivamente, no ano de 2020, enquanto o índice de mortalidade em 2019 ficou em 121.686 nos homens e nas mulheres em 110.344 (INCA, 2021).

Os tratamentos dessa patologia podem ser cirúrgico, radioterápico e quimioterápico, de forma única ou combinada (Zugazagoitia et al., 2016). A quimioterapia é definida como uma administração de substâncias químicas para indução de apoptose, podendo ser administrada via oral, endovenosa, subcutânea, intramuscular ou tópica, sendo classificada como imunoterapia, quimioterapia alvo e a do tipo convencional (Acharya et al., 2017).

A terapia convencional é a administração de um ou mais fármacos por via endovenosa, que atuam de forma sistêmica em diversas etapas do metabolismo celular, seja a célula tumoral ou saudável, mas com o objetivo da destruição do tipo maligna (Poltronieri \& Tusset, 2016). Os medicamentos quimioterápicos capazes de gerar alteração no paladar são: 5fluoruracila (5-FU), leucovorine, oxaliplatina, gencitabine, capecitabine, cisplatina, ciclofosfamida, doxorubicina, irinotecan e metotrexato (Miller et al., 2019).

Devido a abrangência ativa tanto em células malignas, quanto em células normais, tais substâncias são consideradas citotóxicas afetando principalmente a pele, mucosa oral, cabelo e unhas. Em mucosa oral, isso fica evidente pelo surgimento da mucosite oral que são caracterizadas por múltiplas ulcerações circunscritas, superficiais e dolorosas na mucosa não queratinizada (Lacouture \& Sibaud, 2018). Além do mais, as alterações no paladar também podem ocorrer em pacientes submetidos a quimioterapia convencional como: hipogeusia que se caracteriza pela reduzida capacidade perceptiva no paladar; disgeusia é a sensação errada de algum alimento; ageusia é a perda completa do paladar e hipergeusia é a sensação exagerada do sabor (Vries et al., 2018).

Com o aumento expressivo dos casos de câncer e, consequentemente, na execução do tratamento quimioterápico, acarretando danos colaterais em região bucal e sensorial no paladar torna-se necessário uma compreensão abrangente sobre o tema para tentativas de mitigação desses efeitos. Tendo como objetivo do presente trabalho de revisar a literatura acerca das alterações do paladar provocadas por 5-FU, leucovorine, oxaliplatina, gencitabine, capecitabine, cisplatina, ciclofosfamida, doxorubicina, irinotecan e metotrexato, sendo medicamentos antineoplásicos convencionais.

\section{Metodologia}

Este estudo foi realizado através do levantamento de dados no período de 2009 até 2021, a partir da literatura científica na base de dados PubMed, Lilacs, Cochrane e Scielo em língua inglesa e espanhola utilizando as palavras antineoplastics, distorted taste, dysgeusia, stomatitis e oral cavity juntamente com os operadores booleanos "and" e "or", de forma a combinar os descritores citados. O critério de fichamento ocorreu a partir da leitura dos resumos no qual foram 
inclusos artigos que aponte informações relevantes sobre a revisão proposta, além do mais, o programa Mendeley foi utilizado na construção desse artigo.

\section{Revisão de Literatura}

O 5- FU é um fármaco quimioterápico utilizado no tratamento de vários tipos de câncer como colorretal, esofágico, gástrico e anal. Seus efeitos adversos incluem fadiga, náusea, perda de apetite, úlceras e sabor metálico em cavidade oral (Gillis \& Eminger, 2020). Ele faz parte da classe quimioterápica conhecida como antimetabólitos, pois está envolvida no processo de inibição da via de construção da Timidina, um dos nucleotídeos que compõe o DNA. Tal sequência é iniciada pela entrada dessa substância em células malignas, ligando ao timidilato sintetase , que interrompe o processo mitótico da célula, resultando na danificação da estrutura do DNA, fazendo com que sua replicação diminua (Gillis \& Eminger, 2020).

Já a capecitabina é um precursor de carbamato de fluoropirimidina administrado por via oral de 5-FU, foi preliminarmente desenvolvida como um agente oral que poderia mimetizar a ação do 5-FU para contornar alguns efeitos colaterais indesejados da terapia (Dooley \& Goa, 1999). Com o objetivo de maior seletividade, a capecitabina foi projetada para ser convertida em 5-FU preferencialmente no local do tumor após via enzimática de três etapas (Aprile et al., 2009).

O mecanismo exato que provoca esta toxicidade da sequência é desconhecido, mas a interação com folato retido intracelular é uma possibilidade. Toxicidades gastrointestinais superiores e inferiores, incluindo náuseas, vômitos, diarreia, mucosite, distúrbios abdominais, dispepsia e anorexia são frequentemente relatadas entre pacientes expostos à capecitabina e podem ocorrer simultaneamente (Keefe, 2004).

A mecânica de forma detalhada que resulta na disgeusia induzida pelo 5-FU ainda não foi elucidada em sua plenitude, mas se sabe que há a inibição da diferenciação e proliferação de células nas papilas gustativas pela ação tóxica direta da droga quimioterápica, além do mais, a deficiência de zinco devido à quelação provacada pelo 5-FU relatada por Fukasawa et al., (2005), pode ajudar a elucidar, pelo menos em parte, a indução da disgeusia por alguns agentes quimioterápicos, pois foi demonstrado que uma grande quantidade de zinco é necessária para que o mecanismo de regeneração das células nas papilas gustativas aconteça, indicando que a deficiência desse mineral pode causar certo comprometimento do sentido gustativo (Fujii et al., 2018).

A oxaliplatina é um outro fármaco quimioterápico utilizado contra o câncer do tipo sólido, tal substância induz distúrbios na percepção do paladar, porém, a forma como isso é desencadeada não é bem esclarecida (Boltong et al., 2012). Segundo Mogi et al., (2021), a oxaliplatina diminuiu a sensibilidade ao sabor doce com alteração na expressão do receptor desse sabor nas papilas circunvaladas em línguas de rato no período de 3 a 7 dias após administração, e a diminuição foi quase completamente recuperada em 14 dias. Esse mesmo estudo comparou o estudo da oxaliplatina lipossomalizada com a do tipo livre, demonstrando na análise imunohistoquímica o aumento de platina/oxaliplatina na lâmina própria não epitelial lingual, resultando na correlação inversamente proporcional da quantidade acumulada com a diminuição da sensibilidade do sabor doce, mas não com a expressão do receptor desse sabor nos níveis nas papilas gustativas no epitélio (Mogi et al., 2021). Além do mais, os nervos gustativos que desencadeiam as sinapses com células gustativas do tipo III nas papilas gustativas que passam pela lâmina própria, transmitindo a sensação do sabor ao sistema nervoso central, reforçando a hipótese de que a platina/oxaliplatina acumulada na lâmina própria pode induzir disfunção dos nervos gustativos, resultando em uma diminuição da sensibilidade ao sabor doce (Ohishi et al., 2016).

Outro fármaco quimioterápico que possui a platina em sua estrutura química é a cisplatina que é um medicamento administrado por via endovenosa, utilizado para tratar pacientes com câncer de bexiga, ovário, região de cabeça e pescoço, pulmão, colo do útero, esôfago, mama, cérebro e testículos (Singh et al., 2018). A condução nervosa avaliada feita antes e após 
o tratamento com tal fármaco mostrou que existem efeitos neurológicos nos pacientes, como perda de audição e no paladar (Aldossary, 2019).

O metotrexato é um outro fármaco quimioterápico antagonista do folato que atua via inibição competitiva da diidrofolato redutase para bloquear o metabolismo do folato, portanto inibe a síntese de DNA e RNA (Neradil et al., 2012). O metotrexato tem sido amplamente utilizado no tratamento de câncer de mama, osteossarcoma e leucemia linfocítica aguda (Sakura et al., 2018). Após infusões dessa droga, a terapia de resgate com ácido folínico (leucovorina) é administrada para reduzir os efeitos colaterais tóxicos (Wennerstrand et al., 2013). A toxicidade do metotrexato inclui toxicidade intestinal, cardiotoxicidade, toxicidade da medula óssea, nefrotoxicidade e hepatotoxicidade limitando o uso dessa droga (Howard et al., 2016; Pivovarov \& Zipursky, 2019). A ação dessa droga nas células normais com a interrupção dos processos metabólicos gerando alteração das vias antioxidantes, antiinflamatórias, apoptóticas e nas expressões de sabor (Huang et al., 2020).

Os receptores de sabor presentes no trato gastrointestinal detectam os principais componentes da dieta e desempenham um papel crítico na determinação das preferências alimentares. Esses receptores estão envolvidos na regulação da ingestão alimentar e na alteração do ambiente microbiano (Avau \& Depoortere, 2016).

A gencitabina é um análogo do nucleosídeo de desoxicitidina cujas propriedades antiproliferativas são dependentes de várias ações inibitórias na síntese de DNA, bloqueando a progressão do ciclo celular no limite da fase G1/S, sendo amplamente utilizada como um agente quimioterápico anticâncer para vários tumores sólidos e alguns linfomas (Wong et al., 2009). Desde 1997, a gencitabina tornou-se a escolha de tratamento padrão para câncer pancreático localmente avançado e metastático (Kim \& Gallick, 2008), entretanto, após semanas de tratamento, tende a desenvolver quimiorresistência de gencitabina em tumores inicialmente sensíveis (Amrutkar \& Gladhaug, 2017). O receptor de sabor amargo da família T2R38 possui um papel importante na biologia do câncer e foi demonstrado recentemente para o câncer de pâncreas (Stern et al., 2018) , e o receptor de sabor T2R10 está relacionado ao câncer de câncer de mama, contudo a suas funções não tenham sido explicadas (Amézaga et al., 2018). Por outro lado, os compostos amargos também podem exibir atividades anticâncer ou intensificadoras da quimioterapia, em que os mecanismos exatos ainda são desconhecidos (Kwatra et al., 2013). A maioria dos receptores amargos são indistintos pois os mecanismos ainda não são bem compreendidos e podem ser desencadeados por diferentes substâncias quimicamente não relacionadas (Stern et al., 2018).

A ciclofosfamida é um agente alquilante, agindo nas células em proliferação, danificando seu DNA e interrompendo a atividade do ciclo celular (Stone et al., 2002). Em condições normais, as células gustativas têm expectativa de vida relativamente curta a depender do tipo de célula, e são continuamente perdidas e substituídas pela camada basal, logo abaixo das papilas gustativas (Feng et al., 2014) onde as células amplificadoras de trânsito sofrem mitose rápida e são alvos naturais para agentes quimioterápicos, como a ciclofosfamida, que anexam fitas abertas de DNA, sugerindo que esse fármaco tem efeito citotóxico no sistema gustativo, agindo nas células gustativas normais, bem como nas células progenitoras no epitélio gustativo, desencadeando alterações no ciclo normal de substituição das células gustativas (Perea-Martinez et al., 2013). Ensaios histológicos de papilas gustativas circunvaladas e fungiformes e glândulas salivares menores puramente serosas sugeriram que a ciclofosfamida tem efeito direto no epitélio gustativo, incluindo a redução do número de papilas fungiformes. No entanto, em uma pesquisa conduzida por Mukherjee et al. 2017, as papilas gustativas circunvaladas não diminuíram em número ou mostraram uma perda de células em até 8-12 dias (Mukherjee et al., 2017).

A doxorrubicina é um antibiótico da classe das antraciclinas amplamente utilizado para o tratamento de vários tipos de câncer, como leucemia aguda, câncer de mama, osso e câncer de ovário (Rizvi et al., 2018). É um fármaco chave na quimioterapia curativa para linfoma de Hodgkin, sarcoma de Ewing e osteossarcomas, com vários efeitos anticâncer (Sunariani et al., 2020). É uma droga citotóxica que desencadeia a formação de espécies radicais reativos de oxigênio (Sunariani et al., 2020). O dano às papilas gustativas devido a radicais reativos de oxigênio ocorre de várias maneiras, uma delas é através da 
diminuição no número de receptores gustativos normais, um aumento no limiar do sabor umami e neurotoxicidade no nervo craniano VII, XI, X que funciona como um nervo sensorial na língua (Si \& Lang, 2018). Danos nas papilas gustativas podem causar gosto metálico em pacientes com câncer associados à quimioterapia com doxorrubicina (Ijpma et al., 2017). As células das papilas gustativas expostas aos oxidantes devido à quimioterapia podem induzir a expressão da Metalotioneína 3 ou MT-3 como uma proteína de defesa do sistema imunológico contra a exposição ao oxidante (Si \& Lang, 2018). O papel de MT-3 para neutralizar a presença de sabor metálico pode ser explicado através da redução dos níveis de radicais reativos de oxigênio no corpo, regulando a homeostase do zinco, que desempenha um papel importante no paladar. Níveis reduzidos desses reativos nas papilas gustativas minimizam o dano aos receptores gustativos, estabilizam o limiar do sabor umami e evita danos às papilas gustativas sensoriais (Rivankar, 2014). O aumento da expressão de MT-3 pode neutralizar a sensação de gosto metálico em pacientes com câncer após a quimioterapia (Ijpma et al., 2016).

O irinotecano é um derivado semi-sintético do alcalóide vegetal camptotecina, conhecido como CPT-11, Camptosar®. É um dos medicamentos antitumorais mais importantes desenvolvidos na última década pois é eficaz contra um amplo espectro de doenças malignas, incluindo carcinomas de cólon, estômago, ovário e pulmão, bem como gliomas malignos (Friedman et al., 2009). No entanto, a administração desse medicamento está associada a vários efeitos colaterais gastrointestinais, como diarreia, dor abdominal, redução do apetite, além de náuseas, vômitos e perda do paladar induzidos pela quimioterapia (Badowski, 2017). As complicações orais, como disfunção da glândula salivar, infecção bacteriana, fúngica ou viral da mucosa, cárie dentária e alterações do paladar, têm sido associadas a uma variedade de agentes quimioterápicos (Vasconcelos et al., 2009).

Foi relatado que a mucosite oral induzida por quimioterapia ocorre em aproximadamente $40 \%$ dos pacientes que recebem drogas quimioterápicas (Hejna et al., 2001). Os achados de microscopia óptica e eletrônica de varredura em um estudo realizado por Ibrahim e Elwan, (2019), demonstraram que a administração de irinotecano causou mudanças estruturais evidentes na mucosa da língua de ratos, essas alterações incluem distorção e atrofia das papilas linguais manifestadas como uma diminuição significativa do comprimento e largura médios das papilas linguais no grupo tratado com irinotecano em comparação com o grupo controle (Ibrahim \& Elwan, 2019). Além disso, os resultados imunohistoquímicos desse mesmo estudo ilustram que o irinotecano diminuiu a proliferação de células da mucosa da língua, conforme indicado pela diminuição do marcador de proliferação na expressão imunohistoquímica Ki67, (Bologna-Molina et al., 2013). Para mais, algumas células epiteliais da mucosa da língua do grupo tratado com irinotecano mostraram sinais de apoptose manifestada por vacuolação citoplasmática, além de algumas alterações nucleares na forma de condensação, picnose e marginação da cromatina. Isso pode ser atribuído ao efeito danificador do DNA do irinotecano, já que o irinotecano inibe a etapa de replicação do DNA da topoisomerase I, a enzima necessária para as células se dividirem e crescerem, induzindo assim quebras de DNA na fita múltipla e, por fim, levando à morte celular por apoptose (Lee et al., 2014).

\section{Resultados e Discussão}

Segundo estudo conduzido por Acharya et al., (2017), uma grande quantidade de pacientes com câncer de mama relataram distúrbios no paladar logo após o início do tratamento quimioterápico, podendo ser justificado pela presença das drogas utilizadas e seus metabólitos na saliva, os quais persistiram por vários dias posteriores a sua administração (Dodde et al., 2003), tais alterações, bem como o surgimento de ulcerações na mucosa, foi associado a quimioterapia com ciclofosfamida, epirrubicina, metotrexato e 5-fluorouracil (Jensen et al., 2008). A amlodipina também pode ser associada a essas mudanças sensoriais gustativas. A concentração de doxorrubicina-ciclofosfamida é descrita na literatura pela ação direta nas células receptoras de sabor (Fox et al., 1987). 
O gosto metálico são recorrentes na literatura, podendo ser considerado como uma mudança no paladar (Mattes, 2011). Segundo estudo qualitativo executado por Boltong et al., (2012), pacientes submetidos a quimioterapia contendo oxaliplatina relataram uma alteração ampla na percepção do sabor e no déficit na sensação do prazer (Boltong et al., 2012). Pacientes em tratamentos quimioterápicos possuem menor predileção em pratos ricos em proteínas, resultando em uma ingestão insuficiente da mesma (Del Fabbro et al., 2017).

As células das glândulas salivares como ductais e acinares são também afetadas pelos fármacos quimioterápicos convencionais que resultam em baixo fluxo salivar, compromentendo a viscosidade da saliva, resultando na sensação de boca seca (Jensen et al., 2008). Segundo Field et al., (1997), as alterações na viscosidade salivar resultam das mudanças química e físicas que influenciam na geração do sinal sensorial percebido como secura, pois a quimioterapia pode danificar estruturas acessórias, como glândulas de Von Ebner e glândulas salivares, causando xerostomia ou boca seca, desencadeando a hipossalivação grave, que pode contribuir para a hipogeusia (Mukherjee et al., 2017)

Segundo o estudo de IJpma et al., (2016), que estudaram pacientes curados de câncer testicular através da quimioterapia utilizando os fármacos etoposídeo e cisplatina com ou sem bleomicina, houve uma alta prevalência de disfunção do paladar, possivelmente devido ao acúmulo de cisplatina no epitélio lingual (Ijpma et al., 2016). Outro estudo conduzido por Vries et al., (2017), descreveram que pacientes com câncer de mama tratados por quimioterapia adjuvante através da combinação de taxanos e antraciclinas relatavam hipogeusia, perda de apetite e boca seca (Vries et al., 2017). Esse mesmo estudo apontou um menor consumo energético nos pacientes que realizaram tratamento antineoplásico, corroborado por Wahnefried \& Peterson et al., (2001).

Uma análise sobre a percepção quimiossensorial gustativa através dos estudos de Postma et al., 2020, apontaram não haver diferenças estatísticas nos testes objetivos com tiras de sabor significativas na função gustativa entre pacientes submetidos a quimioterapia e o grupo de comparação aos quais não receberam essas tiras. Porém, a percepção subjetiva do paladar foi considerada ruim pelos pacientes durante a execução do tratamento quimioterápico do que por aqueles que não se submeteram a tal processo (Postma et al., 2020). Em um estudo realizado por Amézaga et al., 2018, a xerostomia é o sintoma mais frequente relatado pelos pacientes submetidos pela quimioterapia e está fortemente associada ao gosto metálico na boca e perda de sabor ou hipogeusia (Amézaga et al., 2018).

Compreender como os fármacos quimioterápicos afetam as células gustativas e os processos bioquímicos referentes a sua renovação celular pode resultar na descoberta ou aperfeiçoamento de novos métodos ou medicamentos que resultem na proteção do maior número possível de células, o que reduziria os efeitos colaterais dessas drogas e de outros agentes citotóxicos, daí a necessidade e importância da realização de novos estudos bioquímicos e farmacológicos sobre o tema.

\section{Considerações Finais}

Os fármacos quimioterápicos convencionais estudados afetam as células gustativas e os processos bioquímicos referentes a sua renovação celular, gerando alterações no paladar como hipogeusia, disgeusia, ageusia e hipergeusia. Embora alguns quimioterápicos já expliquem sua ação no processo de obtenção do paladar, contudo, não há uma droga descrita para reverter este sintoma, gerando desconforto nos pacientes em tratamento. Sugere-se a importância no desenvolvimento de mais estudos com a presente temática, objetivando uma melhora na qualidade de vida dos indivíduos.

\section{Referências}

Acharya, S., Pai, K. M., Bhat, S., Mamatha, B., Bejadi, V. M., \& Acharya, S. (2017). Oral changes in patients undergoing chemotherapy for breast cancer. Indian Journal of Dental Research, 28(3), 261-268. https://doi.org/10.4103/ijdr.IJDR_379_16 
Aldossary, S. A. (2019). Review on pharmacology of cisplatin: Clinical use, toxicity and mechanism of resistance of cisplatin. Biomedical and Pharmacology Journal, 12(1), 7-15. https://doi.org/10.13005/bpj/1608

Amézaga, J., Alfaro, B., Ríos, Y., Larraioz, A., Ugartemendia, G., Urruticoechea, A., \& Tueros, I. (2018). Assessing taste and smell alterations in cancer patients undergoing chemotherapy according to treatment. Supportive Care in Cancer, 26(12), 4077-4086. https://doi.org/10.1007/s00520-018-4277-z

Amrutkar, M., \& Gladhaug, I. P. (2017). Pancreatic cancer chemoresistance to gemcitabine. Cancers, 9(11), 1-23. https://doi.org/10.3390/cancers9110157

Aprile, G., Mazzer, M., Moroso, S., \& Puglisi, F. (2009). Pharmacology and therapeutic efficacy of capecitabine: Focus on breast and colorectal cancer. AntiCancer Drugs, 20(4), 217-229. https://doi.org/10.1097/CAD.0b013e3283293fd4

Avau, B., \& Depoortere, I. (2016). The bitter truth about bitter taste receptors: Beyond sensing bitter in the oral cavity. Acta Physiologica, 216(4), 407-420. https://doi.org/10.1111/apha.12621

Badowski, M. E. (2017). A review of oral cannabinoids and medical marijuana for the treatment of chemotherapy-induced nausea and vomiting: a focus on pharmacokinetic variability and pharmacodynamics. Cancer Chemotherapy and Pharmacology, 80(3), 441-449. https://doi.org/10.1007/s00280-017-3387-5

Bologna-Molina, R., Mosqueda-Taylor, A., Molina-Frechero, N., Mori-Estevez, A. D., \& Sánchez-Acuña, G. (2013). Comparison of the value of PCNA and Ki-67 as markers of cell proliferation in ameloblastic tumors. Medicina Oral, Patologia Oral y Cirugia Bucal, 18(2). https://doi.org/10.4317/medoral.18573

Boltong, A., Keast, R., \& Aranda, S. (2012). Experiences and consequences of altered taste, flavour and food hedonics during chemotherapy treatment. Supportive Care in Cancer, 20(11), 2765-2774. https://doi.org/10.1007/s00520-012-1398-7

de Vries, Y. C., van den Berg, M. M. G. A., de Vries, J. H. M., Boesveldt, S., de Kruif, J. T. C. M., Buist, N., Haringhuizen, A., Los, M., Sommeijer, D. W., Timmer-Bonte, J. H. N., van Laarhoven, H. W. M., Visser, M., Kampman, E., \& Winkels, R. M. (2017). Differences in dietary intake during chemotherapy in breast cancer patients compared to women without cancer. Supportive Care in Cancer, 25(8), 2581-2591. https://doi.org/10.1007/s00520-017-3668-X

de Vries, Y. C., Winkels, R. M., van den Berg, M. M. G. A., de Graaf, C., Kelfkens, C. S., de Kruif, J. T. C. M., Göker, E., Grosfeld, S., Sommeijer, D. W., van Laarhoven, H. W. M., Kampman, E., \& Boesveldt, S. (2018). Altered food preferences and chemosensory perception during chemotherapy in breast cancer patients: A longitudinal comparison with healthy controls. Food Quality and Preference, 63, 135-143. https://doi.org/10.1016/j.foodqual.2017.09.003

Del Fabbro, E., Orr, T. A., \& Stella, S. M. (2017). Practical approaches to managing cancer patients with weight loss. Current Opinion in Supportive and Palliative Care, 11(4), 272-277. https://doi.org/10.1097/SPC.0000000000000300

Demark-Wahnefried, W Peterson, B. L., Winer, E. P., Marks, L., Aziz, N., Marcom, P. K., Blackwell, K., \& Rimer, B. K. (2001). Changes in weight, body composition, and factors influencing energy balance among premenopausal breast cancer patients receiving adjuvant chemotherapy. Journal of Clinical Oncology, 19(9), 2381-2389.

Dodde, W. I. W., Maring, J. G., Hendriks, G., Wachters, F. M., Groen, H. J. M., De Vries, E. G. E., \& Uges, D. R. A. (2003). Determination of epirubicin and its metabolite epirubicinol in saliva and plasma by HPLC. Therapeutic Drug Monitoring, 25(4), 433-440. https://doi.org/10.1097/00007691-200308000-00003

Dooley, M., \& Goa, K. L. (1999). Capecitabine. 58(1), 69-76.

Feng, P., Huang, L., \& Wang, H. (2014). Taste bud homeostasis in health, disease, and aging. Chemical Senses, 39(1), 3-16. https://doi.org/10.1093/chemse/bjt059

Field, E. A., Longman, L. P., Bucknall, R., Kaye, S. B., Higham, S. M., \& Edgar, W. M. (1997). The establishment of a xerostomia clinic: A prospective study. British Journal of Oral and Maxillofacial Surgery, 35(2), 96-103. https://doi.org/10.1016/S0266-4356(97)90683-5

Fox, P. C., Busch, K. A., \& Baum, B. J. (1987). Subjective reports of xerostomia and objective measures of salivary gland performance. Journal of the American Dental Association (1939), 115(4), 581-584. https://doi.org/10.1016/S0002-8177(87)54012-0

Friedman, H. S., Prados, M. D., Wen, P. Y., Mikkelsen, T., Schiff, D., Abrey, L. E., Yung, W. K. A., Paleologos, N., Nicholas, M. K., Jensen, R., Vredenburgh, J., Huang, J., Zheng, M., \& Cloughesy, T. (2009). Bevacizumab alone and in combination with irinotecan in recurrent glioblastoma. Journal of Clinical Oncology, 27(28), 4733-4740. https://doi.org/10.1200/JCO.2008.19.8721

Fujii, H., Hirose, C., Ishihara, M., Iihara, H., Imai, H., Tanaka, Y., Matsuhashi, N., Takahashi, T., Yamaguchi, K., Yoshida, K., \& Suzuki, A. (2018). Improvement of dysgeusia by polaprezinc, a Zinc-L-Carnosine, in outpatients receiving cancer chemotherapy. Anticancer Research, 38 (11), 6367-6373. https://doi.org/10.21873/anticanres.12995

Fukasawa, T., Orii, T., Tanaka, M., Yano, S., Suzuki, N., \& Kanzaki, Y. (2005). Statistical approach to the drug-induced taste disorders based on zinc chelating ability. Yakugaku Zasshi, 125(4), 377-387. https://doi.org/10.1248/yakushi.125.377

Gillis, A., \& Eminger, L. (2020). Hypogeusia and hyposmia with topical 5-fluorouracil treatment. JAAD Case Reports, 6(7), 650-651. https://doi.org/10.1016/j.jdcr.2020.04.023

Hejna, M., Köstler, W. J., Raderer, M., Steger, G. G., Brodowicz, T., Scheithauer, W., Wiltschke, C., \& Zielinski, C. C. (2001). Decrease of duration and symptoms in chemotherapy-induced oral mucositis by topical GM-CSF: Results of a prospective randomised trial. European Journal of Cancer, 37(16), 19942002. https://doi.org/10.1016/S0959-8049(01)00132-0

Howard, S. C., McCormick, J., Pui, C., Buddington, R. K., \& Harvey, R. D. (2016). Preventing and Managing Toxicities of High-Dose Methotrexate. The Oncologist, 21(12), 1471-1482. https://doi.org/10.1634/theoncologist.2015-0164

Huang, X., Fang, Q., Rao, T., Zhou, L., Zeng, X., Tan, Z., Chen, L., \& Ouyang, D. (2020). Leucovorin ameliorated methotrexate induced intestinal toxicity via modulation of the gut microbiota. Toxicology and Applied Pharmacology, 391(February), 114900. https://doi.org/10.1016/j.taap.2020.114900

Ibrahim, M. A. A. H., \& Elwan, W. M. (2019). Effect of irinotecan on the tongue mucosa of juvenile male albino rat at adulthood. International Journal of Experimental Pathology, 100(4), 244-252. https://doi.org/10.1111/iep.12333 
IJpma, I., Renken, R. J., Gietema, J. A., Slart, R. H. J. A., Mensink, M. G. J., Lefrandt, J. D., Ter Horst, G. J., \& Reyners, A. K. L. (2016). Taste and smell function in testicular cancer survivors treated with cisplatin-based chemotherapy in relation to dietary intake, food preference, and body composition. Appetite, 105, 392-399. https://doi.org/10.1016/j.appet.2016.06.010

IJpma, I., Timmermans, E. R., Renken, R. J., Ter Horst, G. J., \& Reyners, A. K. L. (2017). Metallic Taste in Cancer Patients Treated with Systemic Therapy: A Questionnaire-based Study. Nutrition and Cancer, 69(1), 140-145. https://doi.org/10.1080/01635581.2017.1250922

INCA. (2021). Estatísticas de câncer. https://www.inca.gov.br/numeros-de-cancer

Jensen, S. B., Mouridsen, H. T., Reibel, J., Brünner, N., \& Nauntofte, B. (2008). Adjuvant chemotherapy in breast cancer patients induces temporary salivary gland hypofunction. Oral Oncology, 44(2), 162-173. https://doi.org/10.1016/j.oraloncology.2007.01.015

Keefe, D. M. K. (2004). Gastrointestinal mucositis: A new biological model. Supportive Care in Cancer, 12(1), 6-9. https://doi.org/10.1007/s00520-003-05509

Kim, M. P., \& Gallick, G. E. (2008). Gemcitabine resistance in pancreatic cancer: Picking the key players. Clinical Cancer Research, 14(5), 1284-1285. https://doi.org/10.1158/1078-0432.CCR-07-2247

Kwatra, D., Venugopal, A., Standing, D., Ponnurangam, S., Dhar, A., Mitra, A., \& Anant, S. (2013). Bitter melon extracts enhance the activity of chemotherapeutic agents through the modulation of multiple drug resistance. Journal of Pharmaceutical Sciences, $102(12)$, $4444-4454$. https://doi.org/10.1002/jps.23753

Lacouture, M., \& Sibaud, V. (2018). Toxic Side Effects of Targeted Therapies and Immunotherapies Affecting the Skin, Oral Mucosa, Hair, and Nails. American Journal of Clinical Dermatology, 19(s1), 31-39. https://doi.org/10.1007/s40257-018-0384-3

Lee, C. S., Ryan, E. J., \& Doherty, G. A. (2014). Gastro-intestinal toxicity of chemotherapeutics in colorectal cancer: The role of inflammation. World Journal of Gastroenterology, 20(14), 3751-3761. https://doi.org/10.3748/wjg.v20.i14.3751

Mattes, R. D. (2011). Accumulating evidence supports a taste component for free fatty acids in humans. Physiology and Behavior, 104(4), 624-631. https://doi.org/10.1016/j.physbeh.2011.05.002

Miller, K. D., Nogueira, L., Mariotto, A. B., Rowland, J. H., Yabroff, K. R., Alfano, C. M., Jemal, A., Kramer, J. L., \& Siegel, R. L. (2019). Cancer treatment and survivorship statistics, 2019. CA: A Cancer Journal for Clinicians, 69(5), 363-385. https://doi.org/10.3322/caac.21565

Mogi, K., Kamiya, I., Makino, A., Hirao, A., Abe, R., Doi, Y., Shimizu, T., Ando, H., Morito, K., Takayama, K., Ishida, T., \& Nagasawa, K. (2021). Liposomalization of Oxaliplatin Exacerbates the Non-Liposomal Formulation-Induced Decrease of Sweet Taste Sensitivity in Rats. Journal of Pharmaceutical Sciences, 000, 1-9. https://doi.org/10.1016/j.xphs.2021.07.004

Mukherjee, N., Pal Choudhuri, S., Delay, R. J., \& Delay, E. R. (2017). Cellular mechanisms of cyclophosphamide-induced taste loss in mice. PLoS ONE, 12(9), 1-23. https://doi.org/10.1371/journal.pone.0185473

Neradil, J., Pavlasova, G., \& Veselska, R. (2012). New mechanisms for an old drug; DHFR- and non-DHFR-mediated effects of methotrexate in cancer cells. Klinicka Onkologie, 25(SUPPL.2).

Ohishi, A., Nishida, K., Yamanaka, Y., Miyata, A., Ikukawa, A., Yabu, M., Miyamoto, K., Bansho, S., \& Nagasawa, K. (2016). Oxaliplatin alters expression of T1R2 receptor and sensitivity to sweet taste in rats. Biological and Pharmaceutical Bulletin, 39(4), 578-586. https://doi.org/10.1248/bpb.b15-00935

Perea-Martinez, I., Nagai, T., \& Chaudhari, N. (2013). Functional Cell Types in Taste Buds Have Distinct Longevities. PLoS ONE, 8(1), 1-9. https://doi.org/10.1371/journal.pone.0053399

Pivovarov, K., \& Zipursky, J. S. (2019). Low-dose methotrexate toxicity. Cmaj, 191(15), E423. https://doi.org/10.1503/cmaj.181054

Poltronieri, T. S., \& Tusset, C. (2016). Impacto do Tratamento do Câncer Sobre o Estado Nutricional de Pacientes oncológicos: Atualização da Literatura. Revista Brasileira de Ciências da Saúde, 20(4), 327-332. https://doi.org/10.4034/rbcs.2016.20.04.10

Postma, E. M., Kok, D. E., de Graaf, C., Kampman, E., \& Boesveldt, S. (2020). Chemosensory perception and food preferences in colorectal cancer patients undergoing adjuvant chemotherapy. Clinical Nutrition ESPEN, 40, 242-251. https://doi.org/10.1016/j.clnesp.2020.09.012

Rahnama, M., Madej-Czerwonka, B., Jastrzębska-Jamrogiewicz, I., \& Jamrogiewicz, R. (2015). Analysis of the influence of parenteral cancer chemotherapy on the health condition of oral mucosa. Wspolczesna Onkologia, 19(1), 77-82. https://doi.org/10.5114/wo.2014.45291

Rivankar, S. (2014). An overview of doxorubicin formulations in cancer therapy. Journal of Cancer Research and Therapeutics, 10(4), 853-858. https://doi.org/10.4103/0973-1482.139267

Rizvi, S. F. A., Tariq, S., \& Mehdi, M. (2018). Anthracyclines: Mechanism of action, Classification, Pharmacokinetics and future - a mini review. International Journal of Biotechnology and Bioengineering, 4(4), 81-85.

Sakura, T., Hayakawa, F., Sugiura, I., Murayama, T., Imai, K., Usui, N., Fujisawa, S., Yamauchi, T., Yujiri, T., Kakihana, K., Ito, Y., Kanamori, H., Ueda, Y., Miyata, Y., Kurokawa, M., Asou, N., Ohnishi, K., Ohtake, S., Kobayashi, Y., ... Naoe, T. (2018). High-dose methotrexate therapy significantly improved survival of adult acute lymphoblastic leukemia: A phase III study by JALSG. Leukemia, 32(3), 626-632. https://doi.org/10.1038/leu.2017.283

Si, M., \& Lang, J. (2018). The roles of metallothioneins in carcinogenesis. Journal of Hematology and Oncology, 11(1), 1-20. https://doi.org/10.1186/s13045018-0645-X

Singh, L., Aldosary, S., Saeedan, A. S., Ansari, M. N., \& Kaithwas, G. (2018). Prolyl hydroxylase 2: a promising target to inhibit hypoxia-induced cellular metabolism in cancer cells. Drug Discovery Today, 23(11), 1873-1882. https://doi.org/10.1016/j.drudis.2018.05.016 
Research, Society and Development, v. 10, n. 14, e589101422467, 2021

(CC BY 4.0) | ISSN 2525-3409 | DOI: http://dx.doi.org/10.33448/rsd-v10i14.22467

Stern, L., Giese, N., Hackert, T., Strobel, O., Schirmacher, P., Felix, K., \& Gaida, M. M. (2018). Overcoming chemoresistance in pancreatic cancer cells: Role of the bitter taste receptor T2R10. Journal of Cancer, 9(4), 711-725. https://doi.org/10.7150/jca.21803

Stone, L. M., Tan, S. S., Tam, P. P. L., \& Finger, T. E. (2002). Analysis of Cell Lineage Relationships in Taste Buds. Journal of Neuroscience, 22(11), 45224529. https://doi.org/10.1523/jneurosci.22-11-04522.2002

Sunariani, J., Rossa, L. M., Yuliati, Y., Diyatri, I., Juliastuti, W. S., \& Oki, A. S. (2020). Immunoreactive scoring of metallothionein-3 (MT-3) expression as an indicator of metallic taste rats which are induced by doxorubicin and antioxidant. Malaysian Journal of Medicine and Health Sciences, 16 (July), $42-46$.

Vasconcelos, N. P. S., Caran, E. M. M., Lee, M. L., Lopes, N. N. F., \& Weiler, R. M. E. (2009). Dental maturity assessment in children with acute lymphoblastic leukemia after cancer therapy. Forensic Science International, 184(1-3), 10-14. https://doi.org/10.1016/j.forsciint.2008.11.009

Wennerstrand, P., Mårtensson, L. G., Söderhäll, S., Zimdahl, A., \& Appell, M. L. (2013). Methotrexate binds to recombinant thiopurine S-methyltransferase and inhibits enzyme activity after high-dose infusions in childhood leukaemia. European Journal of Clinical Pharmacology, 69(9), 1641-1649. https://doi.org/10.1007/s00228-013-1521-9

Wong, A., Soo, R. A., Yong, W. P., \& Innocenti, F. (2009). Clinical pharmacology and pharmacogenetics of gemcitabine. Drug Metabolism Reviews, 41(2), 77-88. https://doi.org/10.1080/03602530902741828

Zugazagoitia, J., Guedes, C., Ponce, S., Ferrer, I., Molina-Pinelo, S., \& Paz-Ares, L. (2016). Current Challenges in Cancer Treatment. Clinical Therapeutics, 38(7), 1551-1566. https://doi.org/10.1016/j.clinthera.2016.03.026 